\title{
Fast Soft Tissue Deformation with Tetrahedral Mass Spring Model for Maxillofacial Surgery Planning Systems
}

\author{
Wouter Mollemans, Filip Schutyser, Johan Van Cleynenbreugel, and \\ Paul Suetens \\ Medical Image Computing (Radiology - ESAT/PSI), Faculties of Medicine and \\ Engineering, University Hospital GasthuisBerg, Herestraat 49, B-3000 Leuven, \\ Belgium
}

\begin{abstract}
Maxillofacial surgery simulation and planning is an extremely challenging area of research combining medical imagery, computer graphics and mathematical modelling. In maxillofacial surgery abnormalities of the skeleton of the head are treat by skull remodelling. Since the human face plays a key role in interpersonal relationships, people are very sensitive to changes to their outlook. Therefore planning of the operation and reliable prediction of the facial changes are very important. Recently, the use of 3D image-based surgery planning systems is more and more accepted in this field. Although the bone-related planning concepts and methods are maturing, prediction of soft tissue deformation needs further fundamental research. In this paper we present a soft tissue simulator that uses a fast tetrahedral mass spring system to calculate soft tissue deformation due to bone displacement in a short time interval. Results of soft tissue simulation for patients who had a maxillofacial surgery are shown. Finally we truly validated the simulation results and compared our method with others.
\end{abstract}

\section{Introduction}

Simulation of the deformation of the facial soft tissues due to bone movement, demands a mathematical model that is able to imitate the behavior of the facial tissues. Various models have been proposed for this simulation.

The Finite Element Method (FEM) [1] 2] is a common and accurate way to compute complex deformations of soft tissue, but conventional FEM has a high computational cost and large memory usage. This makes FEM models inappropriate for realtime simulation. Hybrid models based on global parameterized deformations and local deformations based on FEM, have been introduced to solve this problem 3]. Most of these methods, however, are only applicable to linear deformations and valid for small displacements. Furthermore they rely on pre-computing the complete matrix system and are therefore unable to cope with topological changes when these occur during simulation.

Mass Spring systems (MSS) 44 5] are widely used to model deformable objects. They are applied to a variety of problems, such as cloth modelling, 
facial animation or real-time deformation. All approaches use models consisting of mass points and springs. The dynamic behavior of these models is simulated by considering forces at mass points.

A classical mass spring model assumes a discrimination of the object into $n$ points $x_{i}$ with mass $m_{i}$. These points are linked by damped springs. The relation between position, velocity and acceleration for point $x_{i}$ at time $t$ is defined as

$$
m_{i} \frac{d^{2} x_{i}(t)}{d t^{2}}+\gamma \frac{d x_{i}(t)}{d t}+F_{i}^{i n t}(t)=-F_{i}^{e x t}(t)
$$

with $\gamma$ denoting a damping factor, $F_{i}^{\text {int }}(t)$ denoting the resulting internal elastic force caused by strains of adjacent springs and $F_{i}^{\text {ext }}(t)$ denoting the sum of external forces, such as gravity or collision reaction forces. Given initial values for $x_{i}(t), v_{i}(t), F_{i}^{i n t}(t)$ and $F_{i}^{e x t}(t)$ at time $t$, various methods are commonly applied to numerically integrate through time.

Excellent results can be achieved by applying these numerical integration methods in order to animate deformable models. However, due to numerical problems and slow convergence, these approaches are not very well suited to estimate the rest position of mass spring systems. In maxillofacial surgery this rest position is more important than the exact animation. In previous work [6] we proposed a fast tetrahedral mass spring model to directly estimate the deformation, without calculating the animation.

In the fast tetrahedral mass spring model the object is represented by a number of tetrahedra. A model point $p_{i}$ is assigned to each vertex and a spring is attached to each edge of the tetrahedra. When a fixed external displacement is applied to some of the points, the new rest position for each point is found by demanding that the total force $F_{i}^{t o t}$ in each point $i$ is minimal. The total force $F_{i}^{\text {tot }}$ in model point $i$ is defined as the sum of forces executed by all springs $j$ that are connected to point $i$.

$$
F_{i}^{t o t}=\sum_{\forall j \in S_{i}} k_{j} *\left(\left|p_{j}-p_{i}\right|-L_{j}\right) * \frac{p_{j}-p_{i}}{\left|p_{j}-p_{i}\right|}
$$

with $S_{i}$ denoting the set of all adjacent springs connected to $p_{i}, L_{j}$ and $k_{j}$ denote the initial length and the spring constant of spring $j$, respectively. As shown in 6] spring constants are determined considering the local mesh geometry and Youngs modulus $E$ of the deformed material.

When comparing this tetrahedral model to a classical mass spring system, one can state some major advantages. Since the model demands that the total force in each soft tissue point is minimal, it is ensured that the outcome of the simulation will be a new rest position for each point. Second as we use a static constraint to find the new rest position, we don't need to know the damping parameter used in a classical mass spring systems. This gives us one parameter less to estimate. Finally our model uses a tetrahedral mesh where classical mass spring systems are layer based [4]. Layer based models consists of several layers with each the same topology: mass points connected by springs to form triangular structures. The consecutive layers are connected by a set of vertical and diagonal inter-layer 
springs. Obtaining an accurate layer based model starting from volumetric data is certainly not straight froward. Tetrahedral meshes on the other hand, are well studied in classical FEM application and software to automatic generate these meshes, is available.

In this work we present our simulation environment using the fast tetrahedral mass spring system. In the first part the different steps needed for simulation are shown. True validation of the outcome of a simulation is very important and was often lacking in previous work, therefore the validation method is discussed in the second part. Finally we present the results of simulation on real patient data and compare our model to a classical FEM based simulator.

\section{The Soft Tissue Simulator}

\subsection{Acquisition and Bone-Related Planning}

When a patient needs to undergo a maxillofacial surgery, pre operatively a CT scan is taken. Out of the CT data we semi-automatic segment the facial soft tissues. First The CT data is treshholded, such that all the soft tissues are segmented. To keep only the outer facial soft tissues, which are important for the soft tissue simulation, a mask is applied to the segmented data. A tetrahedral mesh, that serves as the input for our soft tissue simulator, is created with the Amira software package 1 .

Maxilin 2 is a 3D bone-related planning system for maxillofacial surgery, originating from development in our group over the last years [7]. This software allows a maxillofacial surgeon to pre operatively determine the necessary bone movements and see the effect of the procedure.

\subsection{Boundary Conditions}

If we want to predict the effect of a maxillofacial surgery we have to map the 3D bone-related planning data to the soft tissues. We call this step the determination of the boundary conditions. To do the mapping we presume that the movement of soft tissue points which join at the skull, is equal to the movement of that part of the skull. This way 3 types of points are defined in our soft tissue model:

- Fixed points: All soft tissue points that join at a part of the skull that will not be moved during surgery, are defined as fixed and are not allowed to move during simulation.

- Joint points: All points that join at a part of the skull that will be displaced during surgery due to distraction or repositioning, are called joint points. From the bone-related planning we know the necessary bone displacement and we can define the necessary displacement of the joint points.

\footnotetext{
${ }^{1}$ http://www.amira.com

${ }^{2}$ http://www.medicim.com
} 

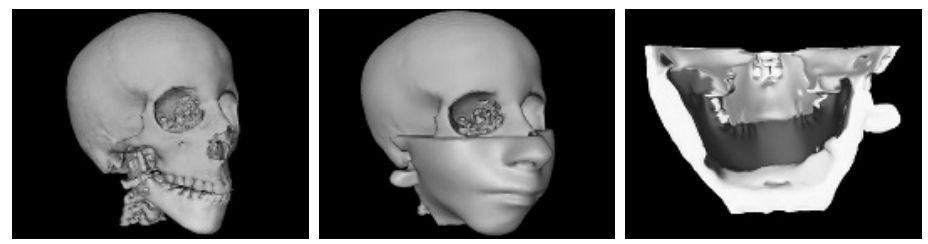

Fig. 1. (a)The planned surgery (created in Maxilim) (b)The planning data is mapped to the soft tissue model (c)Soft tissue model showing the different point types: fixed points (grey), joint points (dark grey) and free points (white).

- Free points: All the other soft tissue points are free. During simulation their movement is completely determined by the resulting force that exists in these points.

The boundary conditions are automatic calculated based on the 3D bonerelated planning and the corresponding soft tissue model. Practice has pointed out that the user sometimes wants to define extra boundary conditions, for example by defining extra fixed points. For this purpose we created a coloring module by which the user can interactively select a point or a group of points and define extra conditions for the selected ones.

\subsection{Simulation}

After preprocessing the patients data, creation of the tetrahedral soft tissue model and determination of the boundary conditions, we can simulate the soft tissue movements due to the bone displacement. The simulator consists of a 3D environment in which the model can be inspected and some interface buttons by which models and boundary conditions can be loaded and simulation can be performed.

It turned out that it is often difficult for a surgeon to immediate see differences between the pre operative and predicted facial skin surface when they are shown together on a workstation. When a dynamic evolution is shown from pre operative to predicted facial surface, the effect of the surgery can much easier be determined. Due to the iterative procedure we use in the fast tetrahedral mass spring system to calculate a new rest position [6], a dynamic transition can easily be generated and shown to the user.

Two main properties for a simulator are its accuracy and the speed of the calculations. Truly validating the accuracy of a simulator is the only way to make a good comparison between two implementations and to see if the approximation goals are reached. Therefore we discuss our validation method in the next section. 


\section{$3 \quad$ Validation Procedure}

During development of a soft tissue model for surgery planning it is very important to measure how close a prediction is to the real post operative result. The only way to truly measure this accuracy is by comparing post operative data with the predicted data.

To obtain the post operative data, four months after surgery the patient gets a new CT scan. This post operative CT scan is rigidly registered to the pre operative CT data, using maximization of mutual information on a unaltered subvolume 8]. From these co-registered post operative data, a surface representation of the skin is generated. This post operative skin surface will be compared to the predicted skin surface.

We distinguish two different techniques to compare the predicted and post operative skin surface: the first is more qualitative, while the second is a quantitative technique. In the first case we let the user visually compare both surfaces and ask him to give a score value between one and ten, where ten indicates a perfect match. With the second procedure 9] we calculate a distance map between the predicted skin surface and the post operative skin surface. This distance map can be projected on the predicted surface by using a color code or by validating the statistics of the error map. When using the color code, dark grey colors mean that large distances between the predicted and pre operative skin surface were found, while a white color symbolizes areas where both surfaces match perfect. This results in an easily interpretable image of the errors over the entire surface

\section{Results}

We show results for three patients suffering from unilateral microsomia. All these patients have been treated with unilateral mandibular distraction. The distraction device had two degrees of freedom: unidirectional translation and angular rotation. Figure 2 presents the bone related planning, the pre operative soft tissue model and the predicted new facial outlook. The pre operative facial contour is shown on top of the predicted facial surface to clarify the difference between both surfaces. Simulations were performed on a standard workstation with a $2 \mathrm{GHz}$ processor and $1 \mathrm{Gbyte}$ of RAM. The soft tissue models contain on average 40000 tetrahedra. All three models were considered as homogenous objects with an elastic modulus $E=3000 P a$.

In their clinical routine, all patients had a post operative CT scan four months after surgery. These post operative images were also employed to validate our predictions by the above described method. The error images derived from differences between the post operative and pre operative skin surface, are shown in figure 3 As can be seen most errors lie in the range of $-3 \mathrm{~mm}$ and $2 \mathrm{~mm}$. We found that there are two main reasons for the prediction error. In the first place, all patients had a fixation bandage during the pre operative CT, soft tissue deformations due to this bandage result in significant errors in the predicted facial surface. Other large errors occur in regions where the bone has been distracted, we are still searching for a method to compensate this effect. 

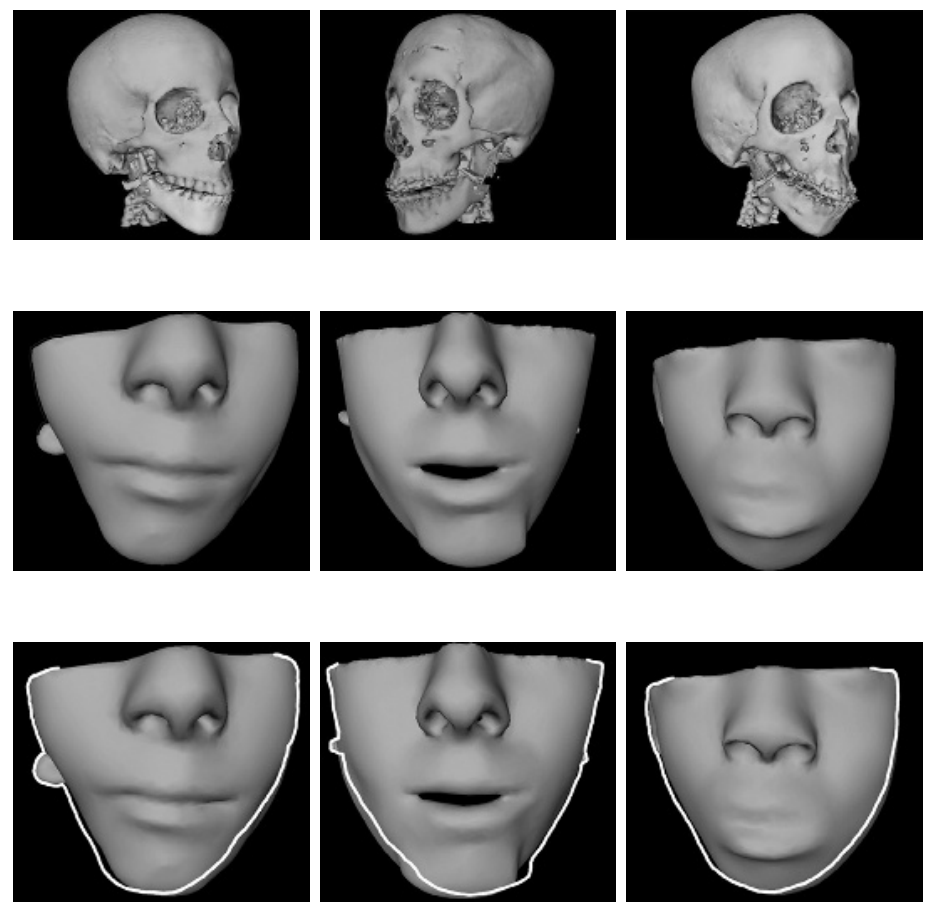

Fig. 2. The pre operative bone-related planning, pre operative facial skin surface and predicted facial surface for the three patients. The pre operative facial contour is shown on top of the predicted facial skin surface.

In the past a FEM based soft tissue simulator has been implemented in our group [2]. To measure accuracy difference between the mass spring system and FEM, we applied the same patient data to the FEM simulator. For the FEM simulations Youngs modulus was chosen at $3000 \mathrm{~Pa}$ and a Poisson coefficient $\nu=$ 0.45 was defined. For both methods the histograms of the error map generated by the above described method, are shown in the second row of figure 3 . We can notice that our simulator based on the mass spring model and the FEM based simulator, generates a very similar result. The model is clearly capable of giving a good approximation of the soft tissue deformation due to bone distraction.

A second important property of a soft tissue simulator is the time needed to make a prediction. As stated in [6] an iterative procedure is used to find the new rest position of all free points after displacement of the joint points. To validate the speed of this procedure we frequently measured the maximal distance between a temporary result, obtained after a certain number of iterations, and the final stable model. The final stable model is the calculated prediction when no point movement could be noticed anymore between to successive iterations. The maximal distance versus time needed, is shown in figure 4 The graph clearly 

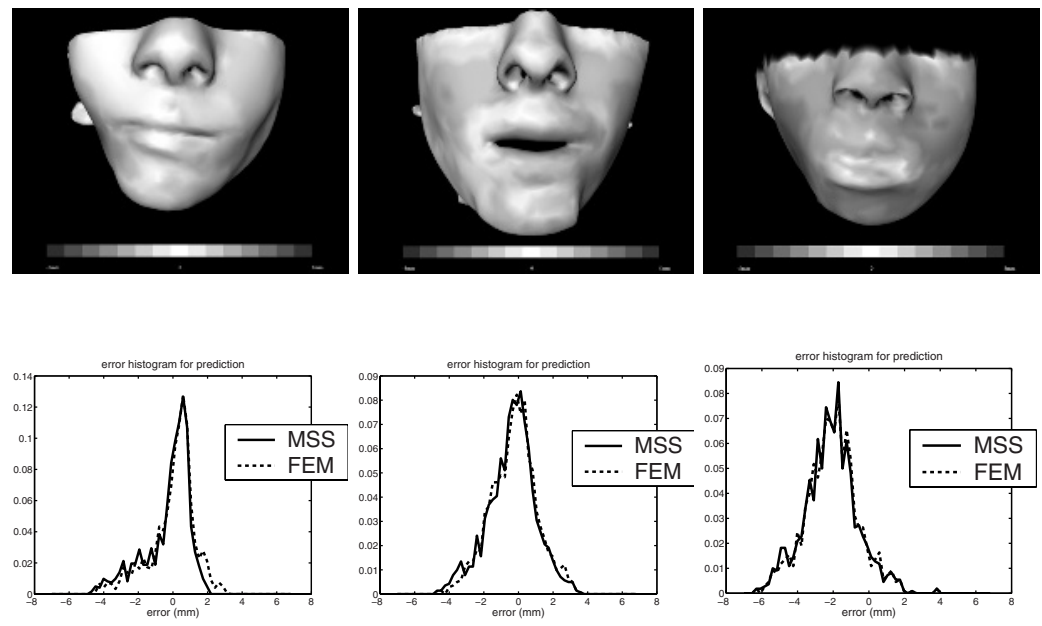

Fig. 3. To validate we measure the distance between predicted and post operative facial skin surface and project these distances on the facial surface with a color code. The scale of the color code ranges from $-8 \mathrm{~mm}$ to $8 \mathrm{~mm}$. A histogram can also be build out of the error data, this is shown in the second row. Histograms of the error data for our simulator that uses the mass spring system and error plots corresponding to deformations calculated with FEM, are shown.

shows that for all three data sets there is an inverse exponential convergence to a stable position. When we take the accuracy of the prediction compared to the real post operative result into consideration, we could state that movements smaller than $1 \mathrm{~mm}$ will have very little influence on the final result. Therefore the iteration procedure can be aborted when maximal movement becomes smaller than $1 \mathrm{~mm}$. The table of figure 4 shows in the first column time needed for each data set to achieve this condition, the second column shows time needed by the FEM based simulator. The maximal and mean distance between the FEM based solution and our solution are shown in column three and four. For our data set, we can conclude that the mass spring system comes much faster to a solution than when the FEM simulator is used. Furthermore the results of both methods are very similar.

\section{Conclusion and Discussion}

We presented our soft tissue simulator that uses a tetrahedral mass spring system to predict the new facial outlook after maxillofacial surgery. An extensive validation procedure was used to truly measure the accuracy of the model and we compared our simulation results with predictions based on a FEM model. We can conclude that our model is faster and at least as accurate as the FEM simulator and gives a good indication of what the face will look like after surgery. 


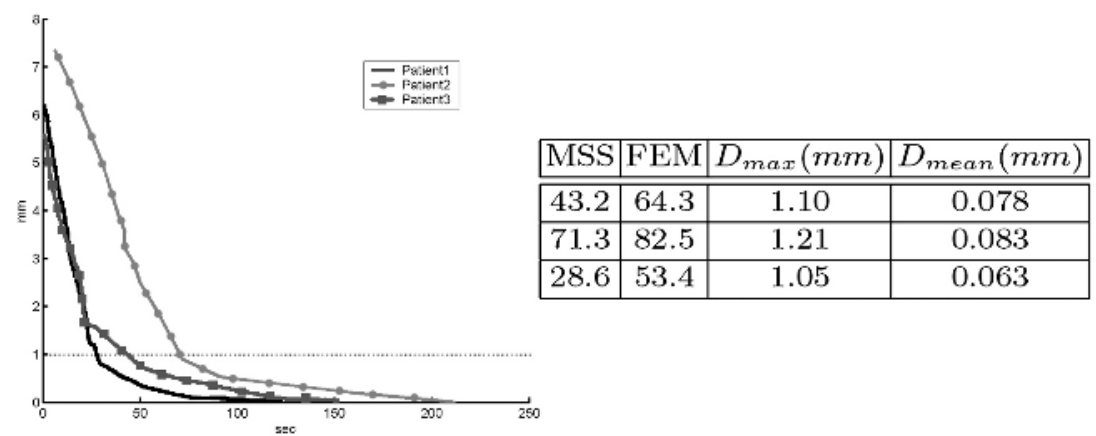

Fig. 4. Left: Maximal distance versus time between the stable prediction and a prediction generated after $n$ seconds. Right: Comparison between the prediction of the MSS and FEM simulator for three patients. The first and second column present time needed for simulation in seconds, the other two columns show maximum and mean distance between both predictions.

However there are still some relative large errors between the predicted and post operative facial outlook. These errors are concentrated around regions where bone was distracted during surgery. Extending our mass spring model so we can compensate for these errors, will be our next challenge. An other field where improvements can be made is determination of the boundary conditions. It would be very useful to know precisely if the soft tissue next to the displaced bone elements stays fixed to it, or what happens exactly. Answers to these questions could influence the prediction result seriously.

Acknowledgements. This work is part of the Flemish government IWT GBOU 020195 project on Realistic image-based facial modelling for forensic reconstruction and surgery simulation and K.U.Leuven/OF/GOA/2004/05.

\section{References}

1. Gladilin, E., Zachow, S., Deuflhard, P., Hege, H.: Towards a realistic simulation of individual facial mimincs. In: SPIE Medical Imaging Conference. (2002)

2. Schutyser, F., Cleynenbreugel, J.V., Ferrant, M., Schoenaers, J., Suetens, P.: Imagebased $3 \mathrm{~d}$ planning for maxillofacial distraction procedures including soft tissue implications. Lecture notes in computer science 1935 (200) 999-1007

3. Muller, M.: Stable real-time deformations. In: ACM SIGGRAPH Symposium on Computer Animation (SCA). (2002)

4. Teschner, M.: Direct Computation of Soft-Tissue Deformation in Craniofacial Surgery Simulation. PhD thesis, Friedrich-Alexander-Universitat (2000)

5. Bourguignon, D., Cani, M.P.: Controlling anisotropy in mass-spring systems. In: The 11th Eurographics Workshop on Animation and Simulation. (2000)

6. Mollemans, W., Schutyse, F., Cleynenbreugel, J.V., Suetens, P.: Tetrahedral mass sping model for fast soft tissue deformation. Lecture notes in computer science $\mathbf{2 6 7 3}$ (2003) 145-154 
7. Poukens, J., Schutyser, F., Cleynenbreugel, J.V., Riediger, D.: 3d planning of distraction osteogenesis with maxilim software. In: Proceedings 4th international congres of maxillofacial and craniofacial distraction. (2003) 251-254

8. Maes, F., Collignon, A., Vandermeulen, D., Marchal, G., Suetens, P.: Multimodality image registration by maximization of mutual information. IEEE transaction on Medical Imaging 16 (1997) 187-198

9. Groeve, P.D., Schutyser, F., Cleynenbreugel, J.V., Suetens, P.: Registration of 3d photographs with spiral ct images for soft tissue simulation in maxillofacial surgery. Lexture notes in computer science 2208 (2001) 991-996 